\title{
Heat Transfer in MHD Micropolar Fluid Flow Past a Vertical Plate in Slip-Flow Regime
}

Ramprakash Sharma * and Abhay Kumar Jha

\begin{abstract}
We consider unsteady flow of a micropolar fluid through a porous medium bounded by a semi-infinite vertical plate in slip-flow regime. A uniform magnetic field acts perpendicular to the porous surface which absorbs the micropolar fluids with a suction velocity varying with time. The free stream velocity follows an exponentially increasing or decreasing small perturbation law. Using approximate method the expression for the velocity microrotation, and temperature are obtained.
\end{abstract}

Keywords: Micropolar fluid, microrotation, slip-flow, magnetohydrodynamics, heat transfer.

\section{Introduction}

Eringen [1, 2, 3] describes some physical systems, which do not satisfy the Navier-Stokes equations. An excellent review of micropolar fluids and their applications was given by Ariman et al. [4]. Gorla et al. [5] have discussed the steady state heat transfer in a micropolar fluid flow over a semi-infinite plate and their analysis is based on the similarity variables. Rees and Pop [6] studied free convection boundary layer flow of a micropolar fluid from a

* Professor of Mathematics, JECRC University, Jaipur, Plot No.IS-2036 to 2039, Ramchandrapura, Sitapura Industrial Area Extn, Near Mahatma Gandhi Hospital , Jaipur-303905, India, rpsharma68@yahoo.com

Received: July 2012, Reviewed: Aug. 2012 
vertical flat plate. Singh [7] have studied free convection flow of micropolar fluid past an infinite vertical plate using finite difference method.

The study of flow and heat transfer for an electrically conducting micropolar fluid past a porous plate under the influence of a magnetic field has attracted the interest of many investigators in view of its applications in many engineering problems such as magnetohydrodynamic (MHD) generators, plasma studies, nuclear reactors, oil exploration, geothermal energy extractions and the boundary layer control in the field of aerodynamics. Also, the porous media heat transfer problems have several practical engineering applications such as crude oil extraction, ground water pollution and another many practical applications such as in biomechanical problems e.g. blood, flow in the pulmonary alveolar sheet and in filtration transpiration cooling. Hiremath and Patil [8] studied the effect on free convection currents on the oscillatory flow of polar fluid through a porous medium, which is bounded by vertical plane surface of constant temperature. Unsteady hydromagnetic free convection flow of Newtonian and polar fluid has been investigated by Helmy [9]. El-Hakien et al. [10] studied the effects of viscous and Joule heating on MHD-free convection flow with variable plate temperature in a micropolar fluid. El-Amin [11] considered the MHD free-convection and mass transfer flow in a micropolar fluid over a stationary vertical plate with constant suction. Kim [12] investigated unsteady free convection flow of micropolar fluid past a vertical plate embedded in porous medium and extended his work [13] to study the effects of heat and mass transfer in MHD micropolar fluid flow past a vertical moving plate. In many practical applications the particle adjacent to a solid surface no longer, takes the velocity of the surface. The particle at the surface has a finite tangential velocity. It slips along the surface. The flow regime is called slip flow regime and this effect cannot be neglected. The study of magneto-micropolar fluid flows in slip flow regime with heat transfer has important engineering applications, e.g. in power generators, refrigeration coils, transmission lines, electric transformers and heating elements. Khandelwal et. al. [14] studied the effects of permeability variation on MHD unsteady flow of polar fluid through a porous medium in slip flow regime over an infinite porous flat plate. Sharma and Chaudhary [15] 180 
studied the effect of variable suction on transient free convective viscous incompressible flow past a vertical plate in slip-flow regime. Recently Sharma [16] investigated the effects of periodic temperature and concentration on unsteady free convection flow past a vertical plate in slip-flow regime.

\section{Mathematical Formulation}

We consider the two-dimensional, unsteady free convective flow of a polar electrically conducting incompressible viscous fluid past a semi-infinite vertical plate embedded in a porous medium and subjected to a transverse magnetic field in the slip-flow regime. It is assumed that the effect of viscous dissipation is negligible in the energy equation. It is also assumed that there is no applied voltage of which implies the absence of an electric field. The transversely applied magnetic field and magnetic Reynolds number are very small and hence the induced magnetic is negligible. It is assumed here that the hole size of the porous plate is significantly larger than a characteristic microscopic length scale of the porous medium. The $\mathrm{x}$-axis is taken along the porous plate in the upward direction and y-axis normal to it. Under these conditions, the governing equations in non dimensional form can be written in a Cartesian frame of reference as

$$
\begin{gathered}
\frac{\partial \mathrm{u}}{\partial \mathrm{t}}-\left(1+\in \mathrm{Ae}^{\delta \mathrm{t}}\right) \frac{\partial \mathrm{u}}{\partial \mathrm{y}}=\frac{\mathrm{d \textrm {U } _ { \infty }}}{\mathrm{dt}}+(1+\beta) \frac{\partial^{2} \mathrm{u}}{\partial \mathrm{y}^{2}}+\mathrm{Gr} \theta \\
+\mathrm{N}\left(\mathrm{U}_{\infty}-\mathrm{u}\right)+2 \beta \frac{\partial \omega}{\partial \mathrm{y}} \\
\frac{\partial \omega}{\partial \mathrm{t}}-\left(1+\in \mathrm{Ae}^{\delta \mathrm{t}}\right) \frac{\partial \omega}{\partial \mathrm{y}}=\frac{1}{\eta} \frac{\partial^{2} \omega}{\partial \mathrm{y}^{2}} \\
\frac{\partial \theta}{\partial \mathrm{t}}-\left(1+\in \mathrm{Ae}^{\delta \mathrm{t}}\right) \frac{\partial \theta}{\partial \mathrm{y}}=\frac{1}{\operatorname{Pr}} \frac{\partial^{2} \theta}{\partial \mathrm{y}^{2}}
\end{gathered}
$$


Ramprakash Sharma and Abhay Kumar Jha

where

$$
\mathrm{N}=\left(\mathrm{M}+\frac{1}{\mathrm{~K}}\right), \eta=\frac{\mu \mathrm{J}^{*}}{\gamma}=\frac{2}{2+\beta} .
$$

The boundary conditions in dimensionless form are

$$
\begin{array}{r}
\mathrm{u}=1+\mathrm{h} \frac{\partial \mathrm{u}}{\partial \mathrm{y}}, \theta=1, \omega=-\mathrm{n} \frac{\partial \mathrm{u}}{\partial \mathrm{y}}, \text { at } \mathrm{y}=0 \\
\mathrm{u} \rightarrow \mathrm{U}_{\infty}, \theta \rightarrow 0, \omega \rightarrow 0, \quad \text { as } \mathrm{y} \rightarrow \infty
\end{array}
$$

\section{Solution}

In order to reduce above system of partial differential equations to a system of ordinary differential equations in dimensionless form, we may represent the translational velocity, microrotation, temperature and concentration as

$$
\begin{aligned}
& u=u_{0}(y)+\in e^{\delta t} u_{1}(y)+0\left(\epsilon^{2}\right) \\
& \omega=\omega_{0}(y)+\in e^{\delta t} \omega_{1}(y)+0\left(\epsilon^{2}\right) \\
& \theta=\theta_{0}(y)+\in e^{\delta t} \theta_{1}(y)+0\left(\epsilon^{2}\right)
\end{aligned}
$$

By substituting the above equations, we obtain following pairs of equations for $\left(\mathrm{u}_{0}, \omega_{0}, \theta_{0}\right)$ and $\left(\mathrm{u}_{1}, \omega_{1}, \theta_{1}\right)$

$$
\begin{gathered}
(1+\beta) \mathrm{u}_{0}^{\prime \prime}+\mathrm{u}_{0}^{\prime}-\mathrm{N} \mathrm{u}_{0}=-\mathrm{N}-\mathrm{Gr} \theta_{0}--2 \beta \omega_{0}^{\prime} \\
(1+\beta) \mathrm{u}_{1}^{\prime \prime}+\mathrm{u}_{1}^{\prime}-(\mathrm{N}+\delta) \mathrm{u}_{1}=-(\mathrm{N}+\delta)-\mathrm{Gr} \theta_{1}-2 \beta \omega_{1}^{\prime}-\mathrm{A} \mathrm{u}_{0}^{\prime} \\
\omega_{0}^{\prime \prime}+\eta \omega_{0}^{\prime}=0
\end{gathered}
$$


Heat Transfer in MHD Micropolar Fluid Flo Mapana J Sci, 11, 3(2012)

$$
\begin{gathered}
\omega_{1}^{\prime \prime}+\eta \omega_{1}^{\prime}-\delta \eta \omega_{1}=-\mathrm{A} \eta \omega_{0}^{\prime} \\
\theta_{0}^{\prime \prime}+\operatorname{Pr} \theta_{0}^{\prime}=0 \\
\theta_{1}^{\prime \prime}+\operatorname{Pr} \theta_{1}^{\prime}-\operatorname{Pr} \delta \theta_{1}=-\mathrm{A} \eta \theta_{0}^{\prime}
\end{gathered}
$$

where the primes denote differentiation with respect to $y$. The corresponding boundary conditions can be written as

$$
\begin{gathered}
\mathrm{u}_{0}=1+\mathrm{h} \frac{\partial \mathrm{u}_{0}}{\partial \mathrm{y}}, \mathrm{u}_{1}=\mathrm{h} \frac{\partial \mathrm{u}_{1}}{\partial \mathrm{y}}, \omega_{0}=-\mathrm{nu}_{0}^{\prime}, \\
\omega_{1}=-\mathrm{n} \mathrm{u}_{1}^{\prime}, \theta_{0}=1, \theta_{1}=0 \text { at } \mathrm{y}=0 \\
\mathrm{u}_{0}=1, \quad \mathrm{u}_{1}=1, \quad \omega_{0}=0, \omega_{1}=0, \theta_{0}=0, \theta_{1}=0 \text { as } \mathrm{y} \rightarrow \infty .
\end{gathered}
$$

The solutions of equations with satisfying boundary conditions are given by

$$
\begin{gathered}
u_{0}(y)=1+B_{6} e^{-h_{1} y}-a_{2} e^{-\operatorname{Pr} y}-a_{3} e^{-h_{6} y}+a_{4} e^{-\eta y} \\
u_{1}=1+d_{14} e^{-h_{2} y}-d_{1} e^{-h_{3} y}+d_{11} e^{-\operatorname{Pr} y}-d_{12} e^{-h_{6} y} \\
+d_{4} e^{-h_{4} y}+d_{5} e^{-h_{5} y}-d_{13} e^{-\eta y}+d_{7} e^{-h_{1} y} \\
\omega_{0}(y)=B_{1} e^{-\eta y} \\
\omega_{1}(y)=B_{2} e^{-h_{5} y}-\frac{A \eta}{\delta} e^{-\eta y} \\
\theta_{0}(y)=e^{-\operatorname{Pr} y}
\end{gathered}
$$




$$
\theta_{1}(\mathrm{y})=\frac{\mathrm{A} \operatorname{Pr}}{\delta}\left(\mathrm{e}^{-\mathrm{h}_{3} \mathrm{y}}-\mathrm{e}^{-\operatorname{Pr} \mathrm{y}}\right)
$$

\section{Results and Discussion}

The formulation of MHD convective flow and heat transfer of an incompressible micro polar fluid along a semi-infinite vertical plate in a porous medium has been carried out in preceding sections. This enables us to carry out the numerical calculations for the distribution of the translational velocity, micro rotation, temperature and concentration across the boundary layer for various values of the parameter. In the present study we have chosen $\mathrm{t}=1, \delta=0.01, \in=0.01$ and $\mathrm{A}=0.5$ while $\mathrm{n}, \beta, \mathrm{M}, \mathrm{Gr}, \mathrm{K}, \mathrm{Pr}$ and $h$ are varied over a range which are listed in figure legends. The effects of viscosity ratio $\beta$ on the translational velocity and the micro rotation profiles across the boundary layer are presented in figures 1 and 2.

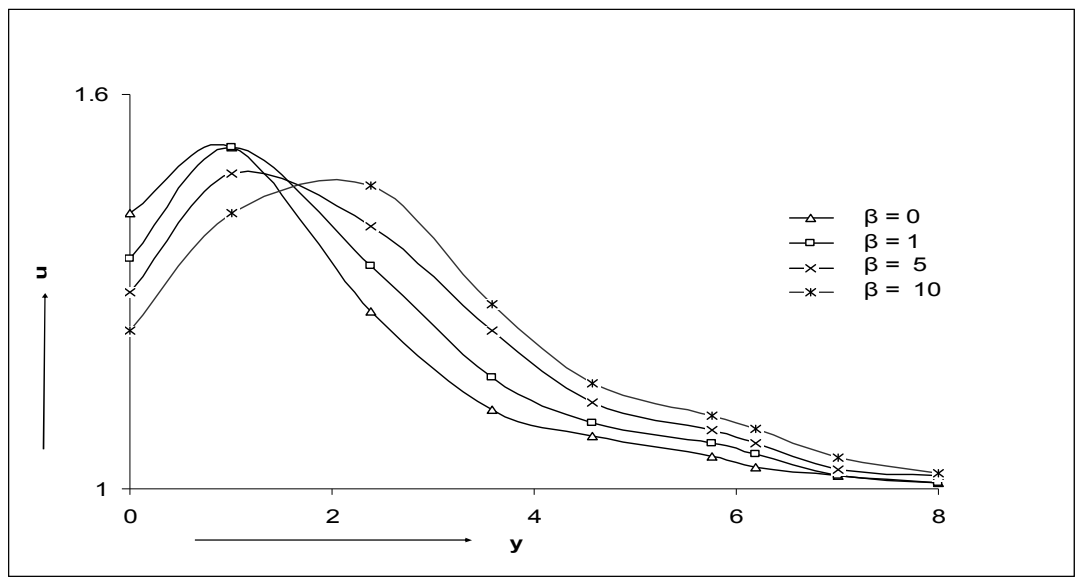

Fig.1 Velocity profiles for various values of $\beta$ with $n=0.5, M=2, K=2$,

$$
\mathrm{Gr}=2, \operatorname{Pr}=1, \mathrm{~h}=0.4, \mathrm{~A}=0.5, \mathrm{t}=1 \text {. }
$$




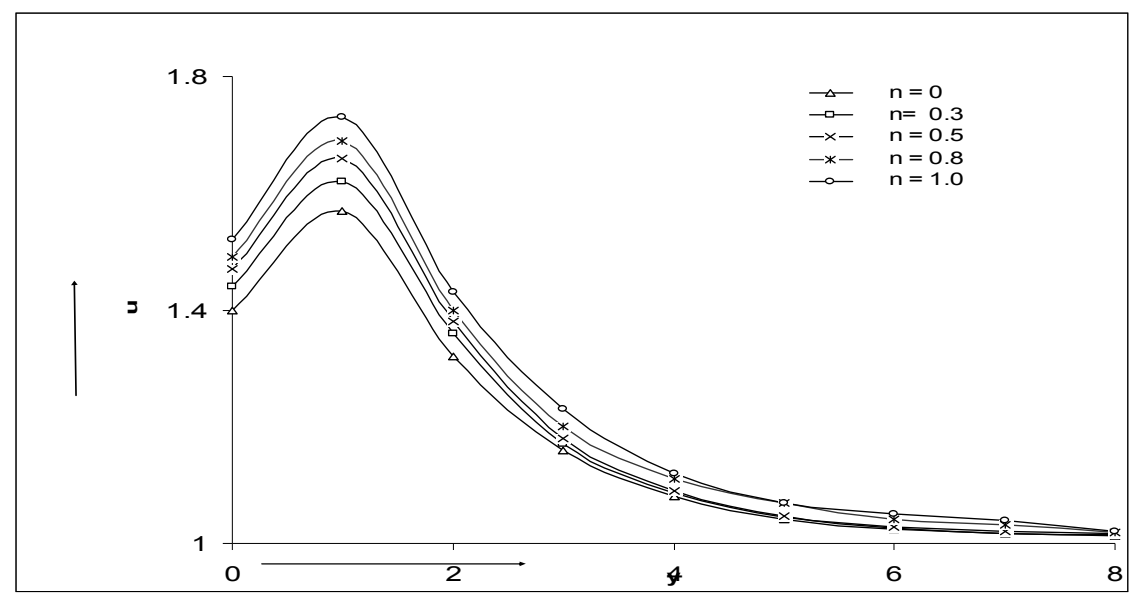

Fig. 2 Velocity profiles for various values of $n$ with $\beta=0.5, M=2, K=2$, $\mathrm{Gr}=2, \operatorname{Pr}=1, \mathrm{~h}=0.4, \mathrm{~A}=0.5, \mathrm{t}=1$.

It is noted that the translational velocity distribution shows a decelerating nature near the plate as $\beta$ increases and then decays to the relevant free stream velocity. In addition the magnitude of microrotation increases at the plate and then decreases away from the plate as $\beta$-parameter increases. Figures 3 and 4 show the effects on n-parameter, which is related to microgyration vector and shear stress, on the translational velocity and the microrotation profiles. It is observed that translational velocity increases with increasing value of $n$-parameter whereas magnitude of micro rotation decreases for increasing value of $\mathrm{n}$. For different values of the magnetic field parameter $\mathrm{M}$, the translational velocity and micro rotation profiles are plotted in figures 5 and 6 . It is obvious that the effect of increasing values of M-parameter results in a decreasing velocity distribution across the boundary layer. The results also show that the magnitude of microrotation on the porous plate is decreased as $\mathrm{M}$ decreases. For various values of the permeability parameter $\mathrm{K}$, the profiles of the translational velocity and the microrotation across the boundary layer are shown in figures 7 and 8. The translational velocity increases for increasing values of permeability parameter, but as $\mathrm{K}$ increases, the micro rotation profiles tend to decrease. Figures 9 and 10 depict the velocity and microtation for different values of refraction parameter $h$. It is observed from the figures that the velocity increases with 
increasing values of parameter $h$ near the plate and starts decreasing away from the plate.

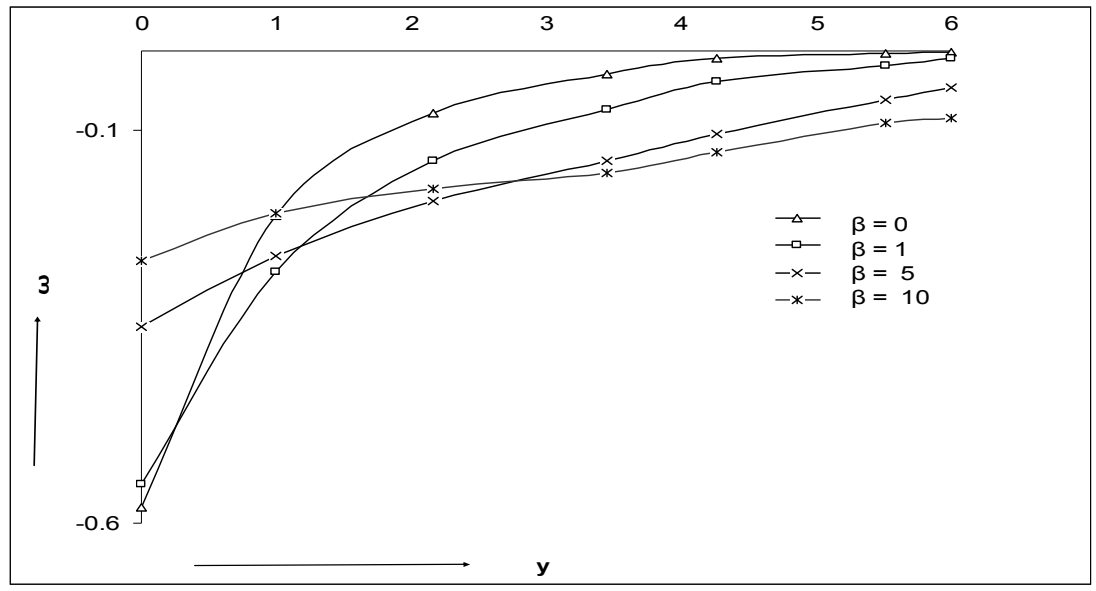

Fig. 3 Micro rotation profiles for various values of $\beta$ with $\mathrm{M}=2, \mathrm{~K}=2, \mathrm{Gr}=2$, $\operatorname{Pr}=1, \mathrm{~h}=0.4, \mathrm{~A}=0.5, \mathrm{t}=1, \mathrm{n}=0.5$.

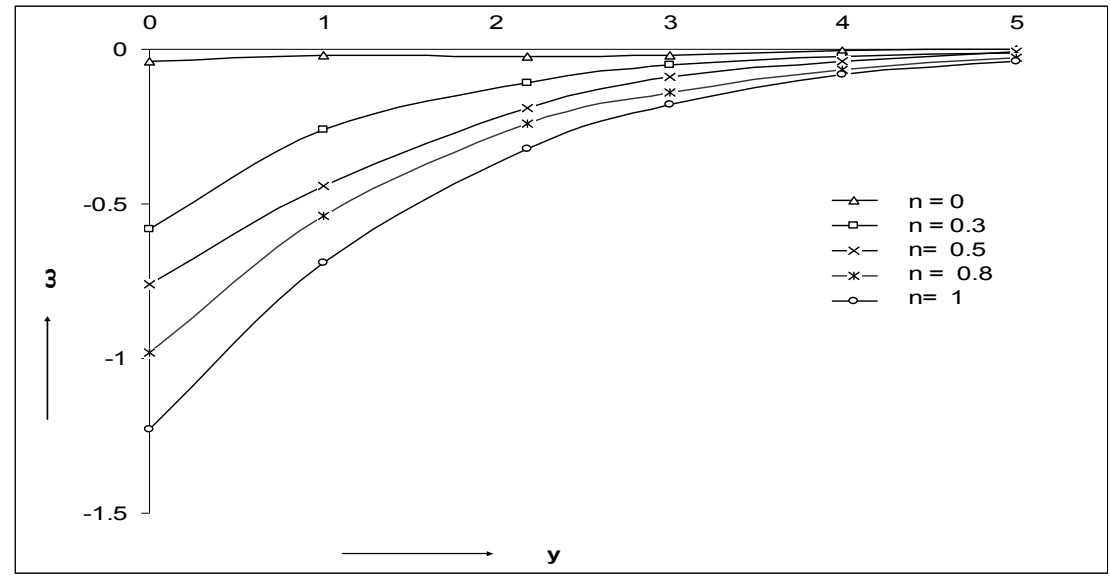

Fig. 4 Micro-rotation profiles for various values of $\mathrm{n}$ with $\beta=.05, \mathrm{M}=2, \mathrm{~K}=2$, $\mathrm{Gr}=2, \operatorname{Pr}=1, \mathrm{~h}=0.4, \mathrm{~A}=0.5, \mathrm{t}=1$. 
Heat Transfer in MHD Micropolar Fluid Flo Mapana J Sci, 11, 3(2012)

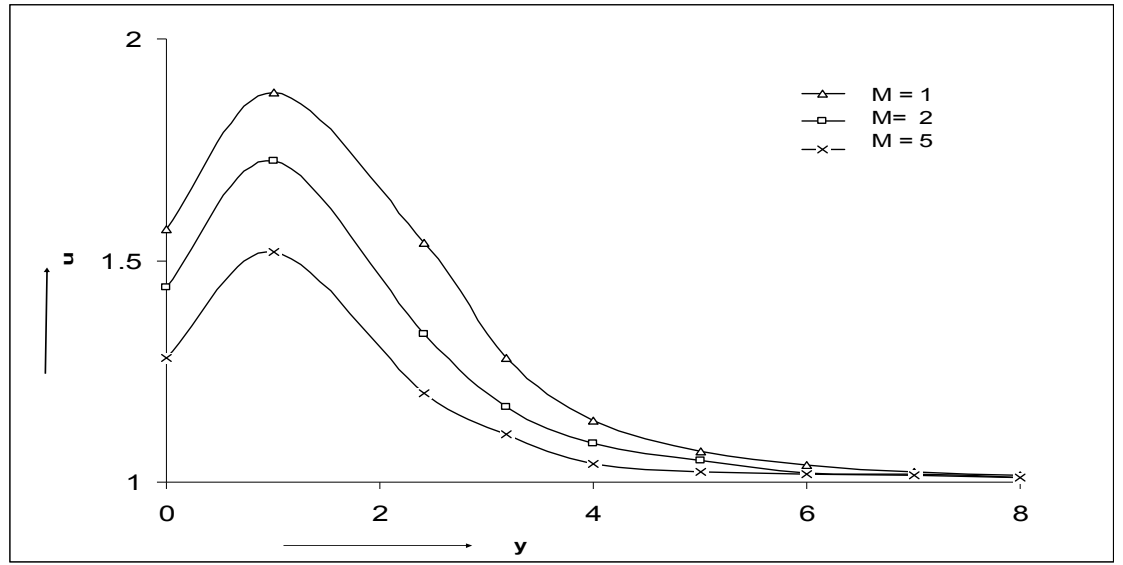

Fig.5 Velocity profiles for various values of $\mathrm{M}$ with $\beta=0.5, \mathrm{n}=0.5, \mathrm{M}=2$, $\mathrm{K}=2, \mathrm{r}=2, \operatorname{Pr}=1, \mathrm{~h}=0.4, \mathrm{~A}=0.5, \mathrm{t}=1$.

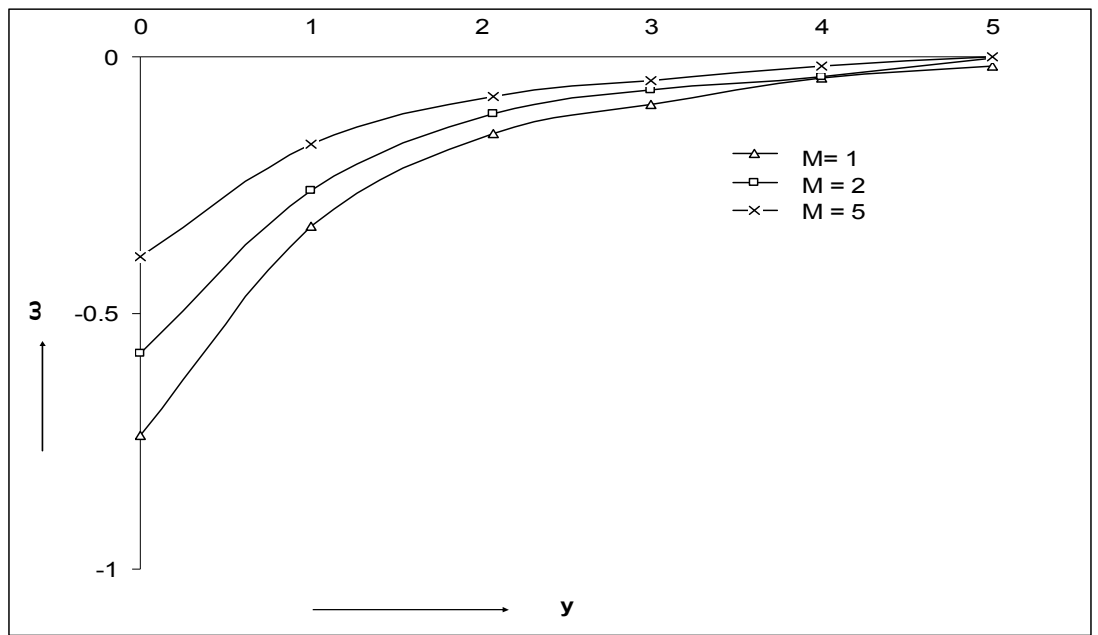

Fig.6 Micro rotation profiles for various values of $\mathrm{M}$ with $\beta=0.5, \mathrm{n}=.05, \mathrm{~K}=2$, $\mathrm{Gr}=2, \operatorname{Pr}=1, \mathrm{~h}=0.4, \mathrm{~A}=0.5, \mathrm{t}=1$. 


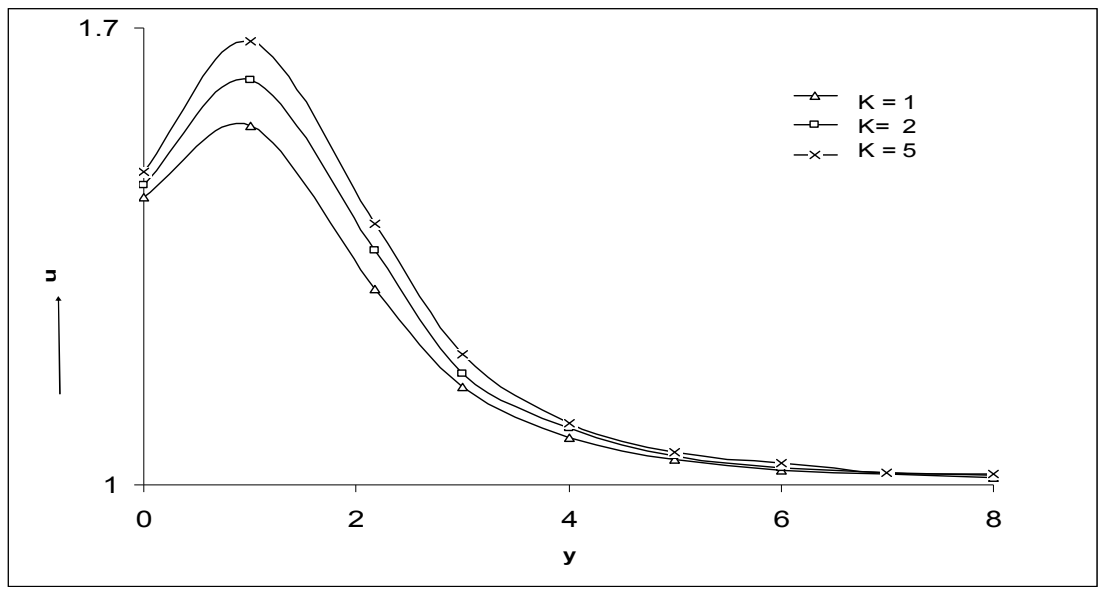

Fig.7 Micro rotation profiles for various values of $\mathrm{K}$ with $\beta=.05, \mathrm{n}=.05, \mathrm{M}=2$, $\mathrm{Gr}=2, \operatorname{Pr}=1, \mathrm{~h}=0.4, \mathrm{~A}=0.5, \mathrm{t}=1$.

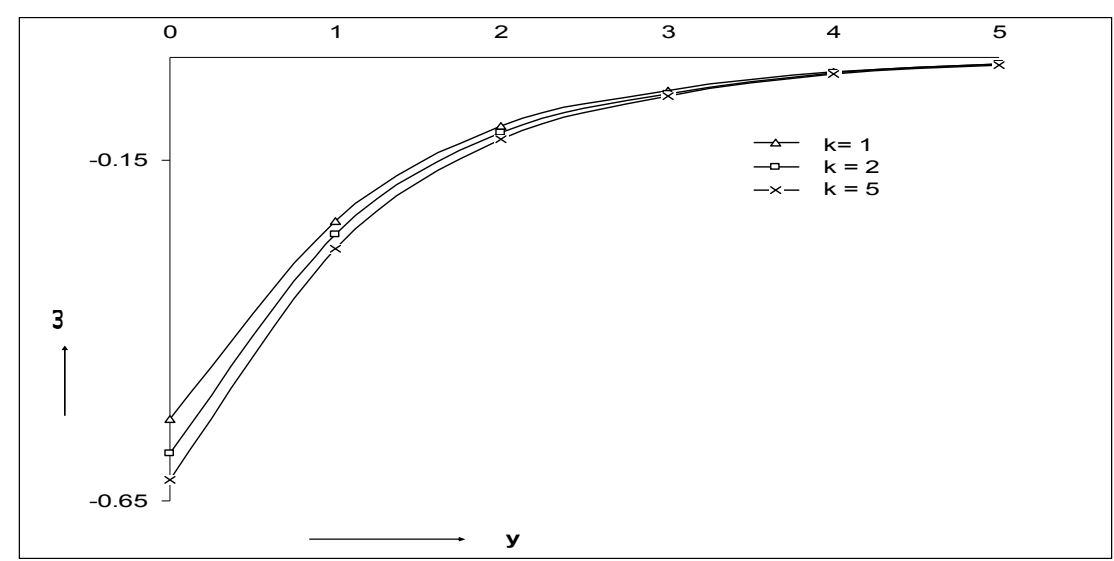

Fig. 8 Micro rotation profiles for various values of $\mathrm{K}$ with $\beta=.05, \mathrm{n}=.05, \mathrm{M}=2$, $\mathrm{Gr}=2, \operatorname{Pr}=1, \mathrm{~h}=0.4, \mathrm{~A}=0.5, \mathrm{t}=1$. 
Heat Transfer in MHD Micropolar Fluid Flo Mapana J Sci, 11, 3(2012)

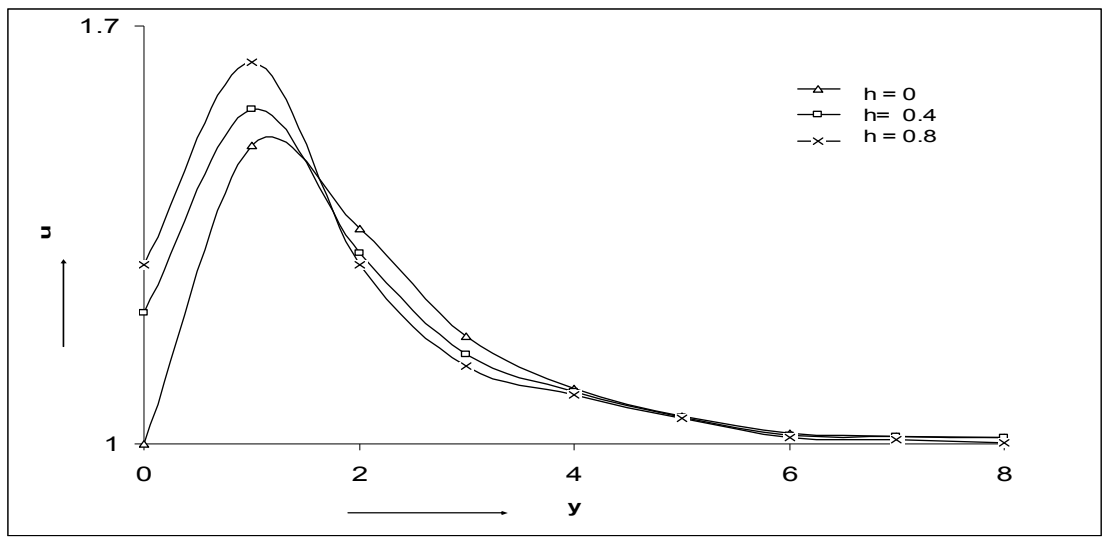

Fig. 9 Velocity profiles for various values of $\mathrm{h}$ with $\beta=0.5, \mathrm{n}=0.5, \mathrm{M}=2$, $\mathrm{Gr}=2, \operatorname{Pr}=1, \mathrm{~K}=2, \mathrm{~A}=0.5, \mathrm{t}=1$.

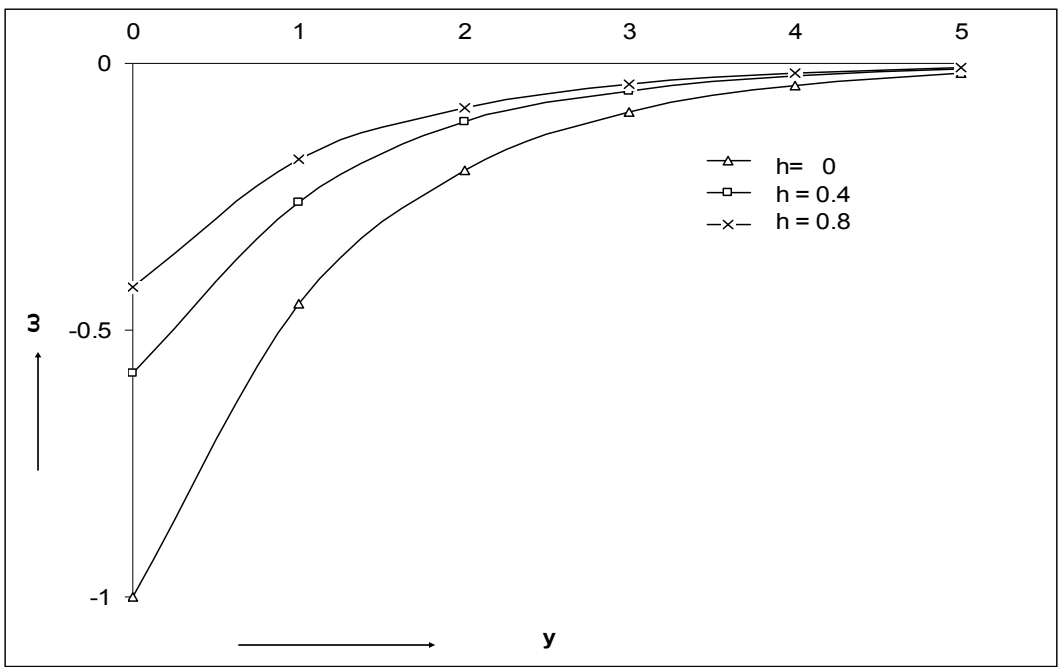

Fig.10 Micro rotation profiles for various values of $\mathrm{h}$ with $\beta=.05, \mathrm{n}=.05, \mathrm{M}=2$, $\mathrm{Gr}=2, \operatorname{Pr}=1, \mathrm{~K}=2, \mathrm{~A}=0.5, \mathrm{t}=1$.

The results also reveal that the micro rotation profiles also increase with increasing values of parameter $h$. The variation of the temperature profiles for different values of Prandtl number Pr have been shown in figure 11 . 


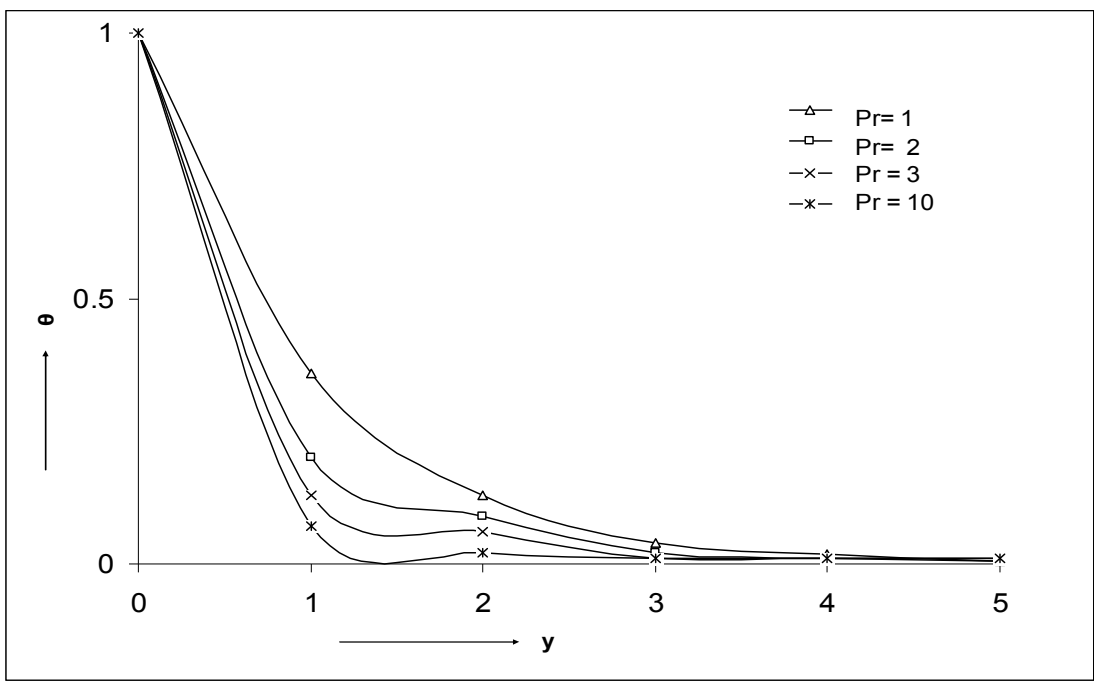

Fig.11 Temperature profiles for various values of $\operatorname{Pr}$ with $\beta=0.5, \mathrm{n}=0.5, \mathrm{M}=2$, $\mathrm{Sc}=1, \mathrm{Gr}=2, \mathrm{~h}=0.4, \mathrm{~K}=2, \mathrm{~A}=0.5, \mathrm{t}=1$.

The results show that an increase of Prandtl number results a decrease in the thermal boundary layer thickness and more uniform temperature distribution across the boundary layer.

\section{References}

[1] A C Eringen, Simple microfluids, Int. J. Engng. Sci., vol. 2, pp. 205-217, 1964.

[2] A C Eringen, Theory of micropolar fluids. J. Math. Mech., vol. 16, pp. 1-18, 1966.

[3] A C Eringen, Theory of thermomicrofluids. J. Math. Anal. Appl., vol. 38, pp. 480-496, 1972.

[4] T Ariman, M A Turk and N D Sylvester, Microcontinuum fluid mechanics - A review, Int. J. Engng. Sci., vol. 11, pp. 905-930, 1973.

[5] R S R Gorla, Mixed convection in a micropolar fluid from a vertical surface with uniform heat flux, Int. J. Engng. Sci., vol. 30, pp. 349-358, 1992.

[6] D A S Rees and I Pop, Free convection boundary layer flow of a micropolar fluid from a vertical flat plate, IMA J. Appl. Math., vol. 61, pp. 179-197, 1998. 
[7] Singh Ajay Kumar, Numerical solution of unsteady free convection flow of an incompressible micropolar fluid past an infinite vertical plate with temperature gradient dependent heat source, J. Energy Heat and Mass Transfer, vol. 24, pp. 185-194, 2002.

[8] P S Hiremath and P M Patil, Free convection effects on oscillatory flow of couple stress field through a porous medium, Acta Mech., vol. 98, pp. 143-158, 1993.

[9] K A Helmy, MHD unsteady free convection flow past a vertical porous plate, ZAMM, vol. 98, pp. 255-270, 1998.

[10] M A El-Hakiem, A A Mohammadein, S M M El-Kabeir and R S R Gorla, Joule heating effects on magnetohydrodynamic free convection flow of a micropolar fluid, Int. Comm. Heat Mass Trans., vol. 26, pp. 219-227, 1999.

[11] M F El-Amin, Magnetohydrodynamic free convection and mass transfer flow in micropolar fluid with constant suction, J. Magn. Mater., vol. 234, pp. 567-574, 2001.

[12] Y J Kim, Unsteady convection flow of micropolar fluids past a vertical plate embedded in a porous medium, Acta. Mech., vol. 148, pp. 105-116, 2001.

[13] Y J Kim, Heat and mass transfer in MHD micropolar flow over a vertical moving plate in a porous medium. Trans. Porous Media, vol. 56, pp. 17-37, 2004.

[14] Khandelwal K Anil, Gupta, Poonam and N C Jain, Effects of couple stresses on the flow through a porous medium with variable permeability in slip flow regime, Ganita, vol. 54, pp. 203-212, 2003.

[15] P K Sharma and R C Chaudhary, Effect of variable suction on transient free convective viscous incompressible flow past a vertical plate with periodic temperature variations in slip flow regime, Emirates Journal of Engineering Research, vol. 8, pp. 33-38, 2003.

[16] P K Sharma, Influence of periodic temperature and concentration on unsteady free convective viscous incompressible flow and heat transfer past a vertical plate in slip-flow regime, Matematicas: vol. XIII, pp. 51-62, 2005. 